\title{
Spectator electric fields, de Sitter spacetime, and the Schwinger effect
}

\author{
Massimo Giovannini* \\ Department of Physics, Theory Division, CERN, 1211 Geneva 23, Switzerland \\ and INFN, Section of Milan-Bicocca, 20126 Milan, Italy
}

(Received 30 January 2018; published 15 March 2018)

\begin{abstract}
During a de Sitter stage of expansion, the spectator fields of different spin are constrained by the critical density bound and by further requirements determined by their specific physical nature. The evolution of spectator electric fields in conformally flat background geometries is occasionally concocted by postulating the existence of ad hoc currents, but this apparently innocuous trick violates the second law of thermodynamics. Such a problem occurs, in particular, for those configurations (customarily employed for the analysis of the Schwinger effect in four-dimensional de Sitter backgrounds) leading to an electric energy density which is practically unaffected by the expansion of the underlying geometry. The obtained results are compared with more mundane situations where Joule heating develops in the early stages of a quasi-de Sitter phase.
\end{abstract}

DOI: 10.1103/PhysRevD.97.061301

Spectator fields are a key element of quantum theories in curved background geometries (see Ref. [1] for two classic monographs on this theme). Their energy density is by definition negligible in comparison with the critical energy density of the background; in the opposite case, they would not spectate the dynamics but instead modify the evolution of the underlying geometry. In the expanding de Sitter spacetime and in some of its inflationary extensions, subcritical fields induce a number of diverse physical effects that further constrain their evolution. Of particular interest have been, in recent years, the spectator gauge fields appearing in different frameworks ranging from magnetogenesis [2] to anisotropic inflation [3]. In the latter context, the spectator fields may become dominant and lead to an explicit breaking of the spatial isotropy by so causing computable (and phenomenologically constrained) anisotropic corrections to the scalar and tensor power spectra [4]. Spectator (electric) fields also appear in the analysis of the Schwinger effect in quasi-de Sitter spacetime [5]; in this context, the constancy of the energy density is achieved by considering a class of homogeneous (electric) field configurations sustained by an appropriate current. This class of spectator fields, superficially allowed in a de Sitter (or quasi-de Sitter) spacetime by analogy with the Minkowskian situation, causes a violation of the second law of thermodynamics.

\footnotetext{
"massimo.giovannini@cern.ch
}

Published by the American Physical Society under the terms of the Creative Commons Attribution 4.0 International license. Further distribution of this work must maintain attribution to the author(s) and the published article's title, journal citation, and DOI. Funded by SCOAP.
The contravariant components of the gauge field strength in conformally flat spacetimes of Friedmann-RobertsonWalker type ${ }^{1}$ are notoriously given by $Y^{i 0}=e^{i} / a^{2}$ and $Y^{i j}=-\epsilon^{i j k} b_{k} / a^{2}$ so that the explicit evolution of the comoving electric (i.e. $\vec{E}=a^{2} \vec{e}$ ) and magnetic (i.e. $\vec{B}=a^{2} \vec{b}$ ) fields can be expressed as

$$
\begin{array}{rlrl}
\vec{E}^{\prime}+4 \pi \vec{J} & =\vec{\nabla} \times \vec{B}, & \vec{B}^{\prime} & =-\vec{\nabla} \times \vec{E}, \\
\vec{\nabla} \cdot \vec{E} & =4 \pi \rho_{q}, & \vec{\nabla} \cdot \vec{B}=0,
\end{array}
$$

where the prime denotes a derivation with respect to the conformal time coordinate $\tau$; the charge density and the comoving current are, respectively, $\rho_{q}$ and $\vec{J}$. Because of Weyl invariance, the well-known form of Eqs. (1) and (2) matches exactly the standard Maxwell equations (see e.g. the first paper of Ref. [2]), but this analogy is misleading since the components of the corresponding canonical energy-momentum tensor are not Weyl invariant. In particular, the electric and magnetic energy densities are diluted as

$$
\rho_{B}(\tau, \vec{x})=\frac{B^{2}(\tau, \vec{x})}{8 \pi a^{4}(\tau)}, \quad \rho_{E}(\tau, \vec{x})=\frac{E^{2}(\tau, \vec{x})}{8 \pi a^{4}(\tau)}
$$

While in Minkowski space the time-independent electromagnetic fields generally lead to a constant energy

\footnotetext{
${ }^{1}$ In the first part of the analysis, the background metric is simply given by $g_{\mu \nu}=a^{2}(\tau) \eta_{\mu \nu} ; \eta_{\mu \nu}=\operatorname{diag}(1,-1,-1,-1)$ is the Minkowski metric, $a(\tau)$ is the scale factor, and $\tau$ denotes the conformal time coordinate. The derivation will be extended to the generally covariant case in the second part of the paper.
} 
density, Eqs. (1), (2), and (3) suggest the opposite: the electromagnetic fields in a conformally flat spacetime of Friedmann-Roberston-Walker type may very well be comovingly constant (i.e. $\vec{E}^{\prime}=\vec{B}^{\prime}=0$ and $\vec{\nabla} \times \vec{E}=$ $\vec{\nabla} \times \vec{B}=0$ ) without leading to a time-independent electromagnetic energy density. Indeed, the simplest gauge configurations compatible with Eqs. (1) and (2) are the ones where i) the fields are comovingly constant [i.e. $\vec{E}(\tau, \vec{x})=\vec{E}_{c}$ and $\vec{B}(\tau, \vec{x})=\vec{B}_{c}$ ], ii) the system is globally neutral (i.e. $\rho_{q}=0$ ), and iii) the total current is absent (i.e. $\vec{J}=0$ ). This is the free adiabatic evolution of the gauge fields; the sources are absent, and the electromagnetic energy densities redshift as $a^{-4}$, becoming quickly negligible as the Universe expands [see Eq. (3)]. This conclusion holds, a fortiori, in the case of a quasi-de Sitter stage of inflationary expansion provided $\vec{E}$ and $\vec{B}$ are bona fide spectator gauge fields. By pursuing the same logic, the solutions of free Maxwell's equations in Minkowskian spacetime can be lifted to the case of a conformally flat background geometry with the important proviso that their energy-momentum tensor is not Weyl invariant. Consequently, if we set the Cauchy data at some initial time $\tau_{i}$ by requiring the electric and magnetic energy densities be smaller than the critical energy density ${ }^{2}$ [i.e. $\rho_{E}\left(\tau_{i}, \vec{x}\right)<3 H^{2} \bar{M}_{\mathrm{P}}^{2}$ and $\rho_{B}\left(\tau_{i}, \vec{x}\right)<3 H^{2} \bar{M}_{\mathrm{P}}^{2}$ ], in the free adiabatic case, the role of gauge fields will be progressively immaterial as the Universe inflates.

The energy densities do not necessarily scale as in the free adiabatic case when the sources are switched on. For instance, in the case of a globally neutral and conducting plasma,

$$
\vec{J}=\sigma \vec{E}, \quad \sigma=a \sigma_{c}, \quad \rho_{q}=0,
$$

where $\sigma$ denotes the comoving conductivity, while $\sigma_{c}$ is commonly referred to as the physical conductivity. If and when the plasma is relativistic, $\sigma_{c}$ is proportional to the physical temperature, and the comoving conductivity is constant as a function of $\tau$. The situation described by Eq. (4) arises during the early stages of a quasi-de Sitter stage of expansion as a possible remnant of a preinflationary epoch (see e.g. the second and third papers of Ref. [2]). Yet a different possibility is that the electric fields are constant in spacetime so that an inhomogeneous solution of Eqs. (1) and (2) is

$$
\begin{aligned}
\vec{\nabla} \times \vec{B} & =4 \pi \vec{J}, & \vec{\nabla} \cdot \vec{B}=\vec{\nabla} \cdot \vec{J}=0, \\
\rho_{B}(\tau, \vec{x}) & =\frac{B^{2}(\vec{x})}{a^{4}(\tau)}, & \rho_{E}(\tau)=\frac{E_{c}^{2}}{a^{4}(\tau)},
\end{aligned}
$$

\footnotetext{
${ }^{2} \mathrm{We}$ shall be using the standard notations implying that the Hubble rate $H=a \mathcal{H}$ where $\mathcal{H}=a^{\prime} / a ;$ moreover, $\bar{M}_{P}=1 / \sqrt{8 \pi G}$.
}

where $E_{c}$ is a constant field and $\vec{B}^{\prime}=0$. Conversely, when the magnetic fields are constant in spacetime, the fully homogeneous electric fields must obey ${ }^{3}$

$$
\begin{aligned}
\vec{E}^{\prime}+4 \pi \vec{J} & =0, \quad \vec{\nabla} \times \vec{B}=\vec{\nabla} \times \vec{E}=0, \\
\rho_{E}(\tau) & =\frac{E^{2}(\tau)}{a^{4}(\tau)}, \quad \rho_{B}(\tau)=\frac{B_{c}^{2}}{a^{4}(\tau)} .
\end{aligned}
$$

The first relation of Eq. (6) suggests that any functional form of the homogeneous electric field can be safely reproduced by postulating the existence of an appropriate comoving current. For the sake of simplicity, the evolution of the electric field can be parametrized as $\vec{E}(\tau)=$ $E_{0} a^{\lambda}(\tau) \hat{n}$ where $E_{0}$ is a constant, $\hat{n}$ is a unit vector, and $\lambda$ is the parameter expressing the evolution of the electric field in units of the underlying rate of evolution. Recalling Eq. (3), the electric energy density will then scale as $\rho_{E} \propto E_{0}^{2} a^{2 \lambda-4}$. Assuming that initially $E_{0}^{2} /\left(H^{2} \bar{M}_{P}^{2}\right) \ll 1$, the critical density bound will always be satisfied at a later time, provided $\lambda \leq 2$. When $\lambda=2$, the electric field is time dependent, but the electric energy density is a spacetime constant; this is exactly the case of Ref. [5], where, more often than not, the contribution of the magnetic field has been neglected by setting, in our notations, $B_{c}=0$. All in all, Eqs. (3) and (6) demonstrate that when the sources do not vanish in Eqs. (1) and (2) the corresponding energy density may be less suppressed than in the free adiabatic case or even become a spacetime constant.

While the chain of arguments presented so far is rarely spelled out in concrete analyses, it is anyway artificial unless the dynamics of the charged species is appropriately discussed. In even simpler words, what about the sources leading to the wanted current? Are they physically sound? When $\lambda \leq 2$, the comoving charge density must vanish, while, according to Eq. (6), the comoving current is given by

$$
\begin{aligned}
\vec{J} & =-\frac{\lambda \mathcal{H}}{4 \pi} a^{\lambda} E_{0} \hat{n} \equiv-\frac{\lambda \mathcal{H}}{4 \pi} \vec{E}, \quad \rho_{q}=0, \\
\mathcal{H} & =a H=\dot{a}
\end{aligned}
$$

A swift comparison of Eqs. (7) and (4) suggests the definition of an effective conductivity $\sigma^{\text {(eff) }}=-\lambda \mathcal{H} /(4 \pi)$ which is negative when the Universe expands (i.e. $\dot{a}>0$ ) and positive when the Universe contracts. ${ }^{4}$ This intuitive analogy already suggests that the current $\vec{J}$ cannot be virtual

\footnotetext{
${ }^{3}$ Note that in Eq. (6) we included $B_{c}$ corresponding to a homogeneous magnetic background; however, this is not necessary for the present discussion so that, in practice, we shall be focusing on the case $B_{c}=0$.

${ }^{4}$ Even if this analogy will not be exploited in the subsequent considerations, it is intuitively useful to appreciate that, in the present case, the expansion rate determines the sign of the effective conductivity.
} 
and should rather arise from the charge carriers entering the total (covariantly conserved) energy-momentum tensor of which the explicit form can be written as

$$
T_{\text {tot }}^{\mu \nu}=T_{\Lambda}^{\mu \nu}+T_{(+)}^{\mu \nu}+T_{(-)}^{\mu \nu}+T_{\text {gauge }}^{\mu \nu},
$$

where $T_{\Lambda}^{\mu \nu}=-p_{\Lambda} g^{\mu \nu}$ denotes the contribution of the cosmological constant and, as usual, $p_{\Lambda}=-\rho_{\Lambda}$. In Eq. (8), $T_{\text {gauge }}^{\mu \nu}$ and $T_{( \pm)}^{\mu \nu}$ are, respectively, the energy-momentum tensors of the gauge field and of the charged species:

$$
\begin{aligned}
T_{\text {gauge }}^{\mu \nu} & =\frac{1}{4 \pi}\left[-Y^{\mu \alpha} Y_{\alpha}^{\nu}+\frac{1}{4} g^{\mu \nu} Y_{\alpha \beta} Y^{\alpha \beta}\right], \\
T_{( \pm)}^{\mu \nu} & =\left(p_{ \pm}+\rho_{ \pm}\right) u_{( \pm)}^{\mu} u_{( \pm)}^{\nu}-p_{ \pm} g^{\mu \nu} .
\end{aligned}
$$

It is well known that the compatibility with the covariant form of Maxwell equations,

$$
\nabla_{\mu} Y^{\mu \nu}=4 \pi j^{\nu}, \quad \nabla_{\mu} \tilde{Y}^{\mu \nu}=0, \quad j^{\nu}=j_{(+)}^{\nu}+j_{(-)}^{\nu},
$$

determines the evolution of the charged components of the energy-momentum tensor; more specifically, we have

$$
\nabla_{\mu} T_{\text {gauge }}^{\mu \nu}=-\left[j_{(+)}^{\alpha}+j_{(-)}^{\alpha}\right] Y_{\alpha}^{\nu}, \quad \nabla_{\mu} T_{( \pm)}^{\mu \nu}=j_{( \pm)}^{\alpha} Y_{\alpha}^{\nu} .
$$

The (covariantly conserved) 4-currents appearing in Eqs. (10) and (11) are, by definition, $j_{( \pm)}^{\alpha}= \pm q \tilde{n}_{ \pm} u_{( \pm)}^{\alpha}$, where $\tilde{n}_{ \pm}$ denote the physical concentration, while $n_{ \pm}=a^{3} \tilde{n}_{ \pm}$are the corresponding comoving concentration. The total comoving charge $\rho_{q}=q\left(n_{+}-n_{-}\right)$vanishes, provided $n_{ \pm}$coincide with a common value (be it $n_{0}$ ). The total comoving current of Eq. (1) is $\vec{J}=q\left[n_{+} \vec{v}_{+}-n_{-} \vec{v}_{-}\right]$, where the 4-velocities appearing in $j_{( \pm)}^{\nu}$ have been expressed as $u_{( \pm)}^{i}=u_{( \pm)}^{0} v_{ \pm}^{i}$ by recalling that $u_{(+)}^{0}=u_{(-)}^{0}=1 / a$. Thanks to the global neutrality of the system, the total current is simply given by $\vec{J}=q n_{0}\left(\vec{v}_{+}-\vec{v}_{-}\right)$. Needless to say, in the case $g_{\mu \nu}=a^{2}(\tau) \eta_{\mu \nu}$, Eq. (10) leads exactly to Eqs. (1) and (2).

Using the explicit expressions of the 4-currents, Eq. (11) becomes, in a conformally flat geometry of FriedmannRobertson-Walker type,

$$
\rho_{ \pm}^{\prime}+3 \mathcal{H}\left(\rho_{ \pm}+p_{ \pm}\right)= \pm \frac{q n_{0}\left(\vec{v}_{ \pm} \cdot \vec{E}\right)}{a^{4}} .
$$

The equations for the positively and negatively charged species can be summed $u^{5}$ by introducing the total

\footnotetext{
${ }^{5}$ The global flow is defined as $(\rho+p) \vec{v}=\left(p_{+}+\rho_{+}\right) \vec{v}_{+}+$ $\left(p_{-}+\rho_{-}\right) \vec{v}_{-}$, where $\vec{v}$ is the total velocity which can be interpreted as the center-of-mass velocity of the charge carriers in the case of massive species. While $\vec{v}$ is related to the sum of the peculiar velocities, the comoving current $\vec{J}$ is given by their difference.
}

energy density (i.e. $\rho=\rho_{+}+\rho_{-}$) and pressure (i.e. $\left.p=p_{+}+p_{-}\right)$:

$$
\rho^{\prime}+3 \mathcal{H}(\rho+p)=\frac{\vec{J} \cdot \vec{E}}{a^{4}}, \quad \vec{J}=q n_{0}\left(\vec{v}_{+}-\vec{v}_{-}\right) .
$$

Since the total current must now be given by the solution of Eq. (7), Eq. (13) becomes

$$
\rho^{\prime}+3 \mathcal{H}(\rho+p)=-\frac{\lambda \mathcal{H}}{4 \pi} E_{0}^{2} a^{2 \lambda-4}
$$

which can be explicitly integrated for any value of the parameter $\lambda$. However, for the sake of simplicity, we shall posit $\lambda=2$ (where the electric energy density turns out to the constant) so that the solution of Eq. (13) becomes, in this case,

$$
\rho(\tau)=\left[\rho\left(\tau_{i}\right)+\frac{E_{0}^{2}}{6 \pi(w+1)}\right]\left(\frac{a_{i}}{a}\right)^{3(w+1)}-\frac{E_{0}^{2}}{6 \pi(w+1)},
$$

where $w=p / \rho$ is the constant barotropic index (i.e. $0 \leq w \leq 1)$ and $\rho\left(\tau_{i}\right)$ denotes the energy density of the charge carriers at the onset of the dynamical evolution, i.e. for an initial time $\tau_{i}$ which might even coincide with the onset of inflation. Equation (15) implies that as long as $a \gg a_{i}$ and $w$ is positive semidefinite $\rho$ violates asymptotically the weak energy condition. This occurrence is prevented, provided the evolution lasts for a limited number of $e$-folds, i.e.

$$
\begin{aligned}
\left(\frac{a_{*}}{a_{i}}\right) & <\left[\frac{6 \pi(w+1) \rho\left(\tau_{i}\right)}{E_{0}^{2}}+1\right]^{\frac{1}{3(w+1)}}, \\
E_{0}^{2} & \ll \rho\left(\tau_{i}\right) \ll 3 H^{2} \bar{M}_{P}^{2} .
\end{aligned}
$$

To fulfil Eq. (16), we must imperatively demand that the electric energy density be smaller in comparison with the (initial) energy density of the charge carriers, i.e. $E_{0}^{2} \ll \rho\left(\tau_{i}\right)$. By recalling that the entropy density is $\varsigma=(\rho+p) / T$, Eq. (13) can be expressed as

$$
\varsigma^{\prime}+3 \mathcal{H} \varsigma=-\frac{4 \mathcal{H}}{T} \rho_{E}
$$

where $\rho_{E}$ is the electric energy density which is constant in the case $\lambda=2$. Equation (17) implies that the entropy fourvector is positive when the background contracts (i.e. $\mathcal{H}<0$ ), and it is negative when the background expands (i.e. $\mathcal{H}>0$ ). Conversely, in the Ohmic case, the second law of thermodynamics corresponding to Eq. (17) stipulates that $\varsigma^{\prime}+3 \mathcal{H} \varsigma=8 \pi \sigma \rho_{E} / T$, and it is always obeyed in spite of the sign of the expansion rate.

Within a fully covariant approach, the gauge field strengths can be decomposed in terms of their covariant 
electric and magnetic components ${ }^{6}$ as argued long ago by Lichnerowicz in the discussion of hydromagnetic nonlinearities [6],

$$
Y_{\mu \nu}=\mathcal{E}_{[\mu} u_{\nu]}+E_{\mu \nu \rho \sigma} u^{\rho} \mathcal{B}^{\sigma}, \quad \tilde{Y}^{\mu \nu}=\mathcal{B}^{[\mu} u^{\nu]}+E^{\mu \nu \rho \sigma} \mathcal{E}_{\rho} u_{\sigma},
$$

where $\mathcal{E}_{[\mu} u_{\nu]}=\mathcal{E}_{\mu} u_{\nu}-\mathcal{E}_{\nu} u_{\mu}$ (and similarly for $\mathcal{B}^{[\mu} u^{\nu]}$ ); note that $\mathcal{E}^{\mu}=Y^{\mu \nu} u_{\nu}$ and $\mathcal{B}^{\mu}=\tilde{Y}^{\mu \nu} u_{\nu}$ generalize, in some sense, the electric and the magnetic components to the relativistic regime. The covariant evolution of the individual energymomentum tensors

$$
\nabla_{\mu} T^{\mu \nu}=Y_{\alpha}^{\nu} j^{\alpha}, \quad \nabla_{\mu} T_{\text {gauge }}^{\mu \nu}=-Y_{\alpha}^{\nu} j^{\alpha}
$$

implies that the contribution of the total current cancels from the sum, i.e. $\nabla_{\mu}\left(T^{\mu \nu}+T_{\text {gauge }}^{\mu \nu}\right)=0$. For the sake of simplicity and for comparison with the noncovariant discussion, the magnetic field will be neglected; in this case, Eq. (19) leads to the following system of covariant equations,

$$
\begin{gathered}
\mathcal{E}^{\mu} \mathcal{E}^{\nu} \sigma_{\mu \nu}-\frac{2}{3} \theta \mathcal{E}_{\alpha} \mathcal{E}^{\alpha}-\frac{1}{2} u^{\mu} \nabla_{\mu}\left(\mathcal{E}_{\alpha} \mathcal{E}^{\alpha}\right)=4 \pi j^{\alpha} \mathcal{E}_{\alpha}, \\
u^{\mu} \nabla_{\mu} \rho+\theta(\rho+p)=-\mathcal{E}^{\alpha} j_{\alpha}
\end{gathered}
$$

where $\sigma_{\mu \nu}$ is the shear tensor, while $\theta=\nabla_{\mu} u^{\mu}$ is the total expansion; both $\theta$ and $\sigma_{\mu \nu}$ appear in the well-known covariant decomposition of $\nabla_{\mu} u_{\nu}$ as

$$
\nabla_{\mu} u_{\nu}=u^{\alpha}\left(\nabla_{\alpha} u_{\nu}\right) u_{\nu}+\sigma_{\mu \nu}+\omega_{\nu \mu}+\frac{\theta}{3} \mathcal{P}_{\nu \mu}
$$

with $\mathcal{P}_{\nu \mu}=g_{\mu \nu}-u_{\mu} u_{\nu}$; and the total relativistic vorticity is antisymmetric for the exchange of the two indices (i.e. $\omega_{\nu \mu}=-\omega_{\mu \nu}$ ) and drops out of Eq. (20) since it is contracted with a term which is symmetric for the same exchange. The covariant analog of the first relation in Eq. (6) can be deduced from Eq. (10), and it is given by

$$
j^{\nu}=\frac{1}{4 \pi} \nabla_{\mu}\left[\mathcal{E}^{\mu} u^{\nu}-\mathcal{E}^{\nu} u^{\mu}\right], \quad \mathcal{B}_{\rho}=0 .
$$

In the case of a conformally flat geometry $g_{\mu \nu}=a^{2}(\tau) \eta_{\mu \nu}$, the dictionary between the various parametrizations of the electric fields is quite straightforward, namely

\footnotetext{
${ }^{6}$ The very notion of relativistic electric or magnetic fields might seem self-contradictory since the electric and magnetic fields are nonrelativistic concepts that must be replaced, in a Lorentz covariant formulation, by the appropriate field strength tensor $Y_{\mu \nu}$ [and by its dual $\left.Y^{\alpha \beta}=\epsilon^{\alpha \beta \mu \nu} Y_{\mu \nu} /(2 \sqrt{-g})\right]$. However, if there exist a family of four-dimensional observers moving with four-velocity $u_{\mu}$, the relativistic dynamics can be phrased in terms of the $\mathcal{E}_{\mu}$ and $\mathcal{B}_{\mu}$ introduced in Eq. (18).
}

$\mathcal{E}^{\mu}=(0, \vec{e} / a)=\left(0, \vec{E} / a^{3}\right) ;$ obviously, $\mathcal{E}_{\mu}=(0,-\vec{e} a)=$ $(0,-\vec{E} / a)$.

The second law of thermodynamics follows by projecting the generally covariant conservation along $u_{\nu}$ :

$$
u_{\nu} \nabla_{\mu} T^{\mu \nu}=Y_{\alpha}^{\nu} j^{\alpha} u_{\nu}
$$

After having introduced the entropy four-vector $\mathcal{S}^{\mu}[7,8]$, Eq. (24) implies that

$$
\nabla_{\mu} \mathcal{S}^{\mu}=\frac{Y_{\alpha}^{\nu} j^{\alpha} u_{\nu}}{T}, \quad \mathcal{S}^{\mu}=\varsigma u^{\mu},
$$

where, from the fundamental identity of thermodynamics, we have $T \varsigma=(\rho+p)$. In the case of the Ohmic conductor, the four-current is given by

$$
j^{\alpha}=\sigma_{\mathrm{c}} Y^{\alpha \beta} u_{\beta} \equiv \sigma_{c} \mathcal{E}^{\alpha},
$$

so that the second law of thermodynamics becomes, in this case,

$$
\nabla_{\mu} \mathcal{S}^{\mu}=\frac{8 \pi}{T} \sigma_{c} \rho_{E} \geq 0, \quad \rho_{E}=-\frac{1}{8 \pi} \mathcal{E}_{\alpha} \mathcal{E}^{\alpha} .
$$

Since $\rho_{E}$ is always positive semidefinite, we also have that $\nabla_{\mu} \mathcal{S}^{\mu} \geq 0$; this is the covariant analog of the Joule heating. In the case of a conformally flat geometry of FriedmannRobertson-Walker type, recalling the dictionary discussed after Eq. (23), we have that $\rho_{E}=E^{2}(\tau) /\left[8 \pi a^{4}(\tau)\right]$; if $\vec{E}(\tau)=$ $E_{0} a^{\lambda} \hat{n}$, we have, in the case $\lambda=2, \rho_{E}=E_{0}^{2} /(8 \pi)$. Recalling that $\nabla_{\mu} \mathcal{S}^{\mu}=\left(\varsigma^{\prime}+3 \mathcal{H} \varsigma\right) / a$, in the noncovariant case, Eq. (27) becomes $\varsigma^{\prime}+3 \mathcal{H} \varsigma=8 \pi \sigma \rho_{E} / T$, where, as already stressed in Eq. (4), $\sigma=\sigma_{c} a$ denotes the comoving conductivity.

When the four-current is determined by Eq. (23), the expression of the second law of thermodynamics follows from Eq. (24), and it can be expressed as

$$
\nabla_{\mu} \mathcal{S}^{\mu}=-\frac{1}{4 \pi T} \mathcal{E}_{\alpha} \nabla_{\beta}\left(\mathcal{E}^{\beta} u^{\alpha}-\mathcal{E}^{\alpha} u^{\beta}\right) .
$$

After some straightforward algebra, the right-hand side of Eq. (28) can be shown to consist of three terms: the first one contains $\left(\nabla_{\beta} u^{\beta}\right) \mathcal{E}_{\alpha} \mathcal{E}^{\alpha}$; the second term is proportional to $\mathcal{E}_{\alpha} u^{\beta} \nabla_{\beta} \mathcal{E}^{\alpha}$; and, finally, the third term contains $\mathcal{E}^{\alpha} \mathcal{E}^{\beta} \nabla_{\beta} u_{\alpha}$. If the covariant decomposition of Eq. (22) is now inserted into Eq. (28), the second law of thermodynamics becomes

$$
\nabla_{\mu} \mathcal{S}^{\mu}=-\frac{4 \theta}{3 T} \rho_{E}-\frac{1}{4 \pi T} \mathcal{E}^{\alpha} \mathcal{E}^{\beta} \sigma_{\alpha \beta}-\frac{u^{\beta}}{T} \nabla_{\beta} \rho_{E},
$$

which is nothing but the generally covariant analog of the first equality reported in Eq. (17), as can be easily verified. Since the shear tensor vanishes when the underlying background is homogeneous and isotropic, Eq. (29) only 
depends upon $\rho_{E}$ and its derivative. But whenever $\rho_{E}$ is covariantly constant, Eq. (29) reduces to

$$
\nabla_{\mu} \mathcal{S}^{\mu}=-\frac{4 \theta}{3 T} \rho_{E}, \quad \theta=\nabla_{\mu} u^{\mu}=3 H
$$

and coincides exactly with Eq. (17) in the conformally flat case. Equation (30) demonstrates that the second law of thermodynamics is only satisfied in the case $H<0$ (i.e. contracting Universe) but not in the expanding case. The general results of Eqs. (27)-(30) also apply when $g_{\mu \nu}(x)=Q(x) \eta_{\mu \nu}$, where $x$ denotes here a generic spacetime point. The pair production due to the Schwinger effect has been evaluated in Ref. [5] by studying the AbelianHiggs model in quasi-de Sitter space, a model already analyzed in the context of inflationary magnetogenesis. In the case of the minimally coupled Higgs field, the largescale magnetic fields are minute, but they are larger in the nonminimally coupled case (see the last paper of Ref. [9]). Among other things, the present analysis demonstrates that the gauge configurations used in Ref. [5] lead to a violation of the second law of thermodynamics when the underlying background geometry expands.

An argument seemingly based on Weyl invariance stipulates that any gauge field configuration is allowed in a conformally flat background geometry, provided the physical currents are appropriately arranged. However, even if the Abelian gauge field equations are Weyl invariant in four spacetime dimensions, the energy-momentum tensor itself is not. Hence, the dilution of the electromagnetic energy density in curved backgrounds cannot be deduced by simply invoking the analogy with the Minkowskian spacetime. In particular, when the Universe expands, the Joule heating is always positive semidefinite, and the second law of thermodynamics does not depend on the sign of the expansion rate. Conversely, the total current needed to maintain a constant (electric) energy density in a curved background can be expressed in terms of an effective conductivity that depends on the expansion rate and on its overall sign. The covariant divergence of the entropy four-vector turns out to be negative semidefinite when the Universe expands, while it is positive semidefinite when the Universe contracts. This observation ultimately causes the violation of the second law in the case of an expanding background where the electric energy density is constant. The conclusions and the bounds obtained from the rate of pair production in an expanding de Sitter spacetime have been derived in the context of a background electric field that violates the second law of thermodynamics. For this reason, they seem neither motivated nor robust. The violations of the second law are instead absent if and when the conformally flat backgrounds contract.
[1] N. D. Birrel and P. C. W. Davies, Quantum Fields in Curved Space (Cambridge University Press, Cambridge, England, 1984); L. Parker and D. Toms, Quantum Field Theory in Curved Spacetime: Quantized Fields and Gravity (Cambridge University Press, Cambridge, England, 2009).

[2] M. Giovannini, Classical Quantum Gravity 23, R1 (2006); Phys. Rev. D 85, 101301 (2012); 86, 103009 (2012).

[3] J. Middleton, Classical Quantum Gravity 27, 225013 (2010); S. Kanno, J. Soda, and M.-a. Watanabe, J. Cosmol. Astropart. Phys. 12 (2010) 024; M. Giovannini, Phys. Rev. D 89, 063512 (2014).

[4] L. Ackerman, S. M. Carroll, and M. B. Wise, Phys. Rev. D 75, 083502 (2007); 80, 069901(E) (2009); A. R. Pullen and M. Kamionkowski, Phys. Rev. D 76, 103529 (2007); M. Giovannini, Phys. Rev. D 93, 043543 (2016).

[5] T. Kobayashi and N. Afshordi, J. High Energy Phys. 10 (2014) 166; C. Stahl, E. Strobel, and S. S. Xue, Phys. Rev. D 93, 025004 (2016); R. Sharma and S. Singh, Phys. Rev. D 96,
025012 (2017); E. Bavarsad, S. P. Kim, C. Stahl, and S. S. Xue, Phys. Rev. D 97, 025017 (2018).

[6] F. de Hoffmann and E. Teller, Phys. Rev. 80, 692 (1950); B. Zumino, Phys. Rev. 108, 1116 (1957); E. Harris, Phys. Rev. 108, 1357 (1957); A. Lichnerowicz, Relativistic Magnetohydrodynamics (Banjamin, New York, 1967); Magnetohydrodynamics: Waves and Shock Waves in Curved Space-Time, (Kluwer, Dordrecht, 1994).

[7] S. Weinberg, Gravitation and Cosmology (Wiley, New York, 1972); L. D. Landau and E. M. Lifshitz, Fluid Mechanics (Pergamon, Oxford, 1987).

[8] L. Tisza, Phys. Rev. 61, 531 (1942); S. Weinberg, Astrophys. J. 168, 175 (1971); S. R. de Groot, V. A. van Leeuwen, and C. G. van Weert, Relativistic Kinetic Theory (North-Holland, Amsterdam, 1980).

[9] M. S. Turner and L. M. Widrow, Phys. Rev. D 37, 2743 (1988); M. Giovannini and M. E. Shaposhnikov, Phys. Rev. D 62, 103512 (2000); M. Giovannini, Phys. Rev. D 95, 083501 (2017). 